\title{
Simultaneous Multiwavelength Observations of the Jets in SS 433
}

\author{
Todd Hillwig* \\ Valparaiso University, USA \\ E-mail: todd.hillwigevalpo.edu \\ Herman Marshall \\ MIT Kavli Institute \\ E-mail: hermanmespace.mit.edu \\ Doug Gies \\ Georgia State University \\ E-mail: gies@chara.gsu.edu
}

\section{Amy Mioduszewski, Michael Rupen}

NRAO

E-mail: amiodusz@nrao.edu, mrupen@aoc.nrao.edu

\begin{abstract}
Simultaneous X-ray, radio, and optical observations have been performed of the microquasar SS 433 in August of 2005. These data have provided an exploration of the jet at several energies and positions. X-ray and optical spectroscopy allows us to explore the agreement between the Doppler shifts, which determines the relative location of the emission along the jet in both wavelength regions. The data also provide information on the jet velocity and any possible changes along the jet trajectory. We discuss the observations and some preliminary results obtained with this data set. Observed unpredicted variability in the Doppler shifts is described and possible scenarios for the changes are considered.
\end{abstract}

VI Microquasar Workshop: Microquasars and Beyond

September 18-22 2006

Società del Casino, Como, Italy

\footnotetext{
* Speaker.
} 


\section{Introduction}

We monitored the jets in the microquasar SS 433 over two weeks in August 2005 with optical spectroscopy, X-ray spectroscopy, and radio imaging. The simultaneous observations allow us to explore tangential motion with proper motions from radio imaging and radial motion through the $\mathrm{X}$-ray and optical spectra. We explore the combination of these data sets in an effort to learn more about the physical motion of the jets.

\section{Observations}

Optical spectra were obtained at the Hobby Eberly Telescope (HET) on Aug. 12, 13, 18, 19 , 22, and 23 and the Kitt Peak National Observatory (KPNO) 2-m on Aug. 15-22, 2006. X-ray spectra were obtained with the Chandra X-ray Observatory on Aug. 6, 12, 16, and 18. Radio images were obtained with the VLBA on Aug. 15-22.

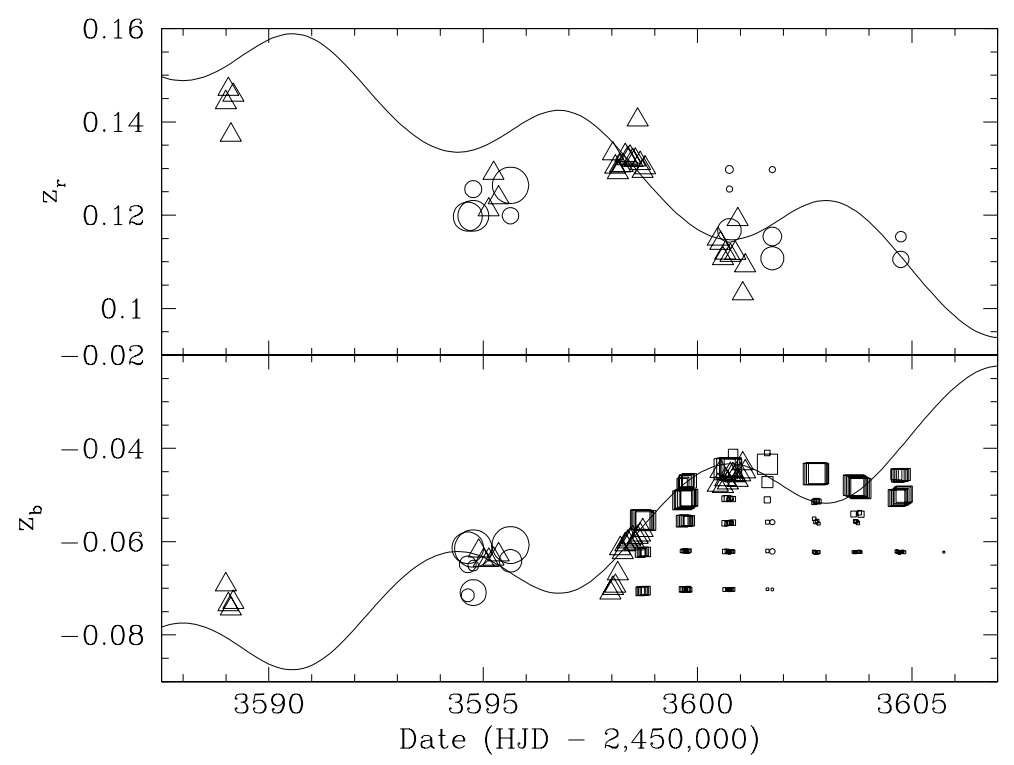

Figure 1: Red- and Blue-shifted jet velocities (in terms of $\mathrm{z}$ ). The symbols denote X-ray data (triangles) as well as optical data from KPNO (squares) and HET (circles). The solid line is the model fit as discussed above. The area of the optical data points is directly proportional to the equivalent width of the jet emission line.

\section{The Jet Emission in SS 433}

Optical emission lines were fit with Gaussian curves. Multiple Gaussians were used only in cases when there were clearly well-separated multiple "bullets" visible. For especially broad lines that were likely overlapping bullets, but were not resolved, a single Gaussian was still used. We used a kinematic model for the jet motion [1] with published parameter fits [2]. There is well known variability in the precession ephemeris. Our data shows an approximate +5 day phase shift 


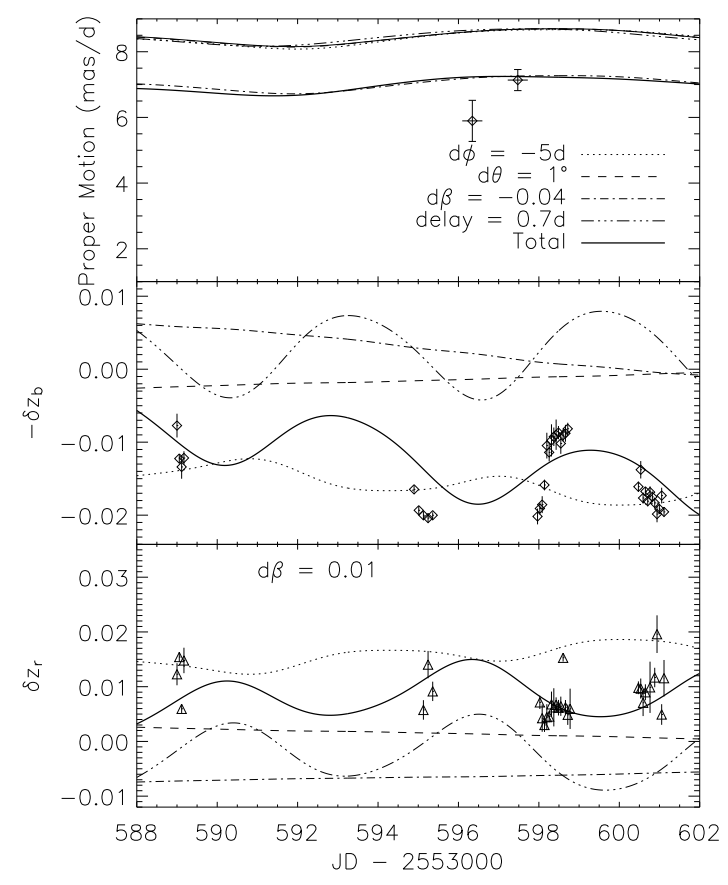

Figure 2: The top frame shows proper motion from radio data, the bottom two frames show residuals and fits to the residuals based on the modified kinematic model [B]].

from the model. Figure 1 shows the X-ray and optical data as well as the model (with a 5.3 day phase shift).

\section{Data Fitting}

The clear deviations between data and model in Figure 1, especially in the red-shifted jet, provide an opportunity to test modifications to the standard precessing, nutating jet model. For example, an anticorrelation has been found between jet speed and jet-cone angle [3]. They provide a modified kinematic model for symmetric jets which describes the jet speed variability. Our data provide an opportunity to test their new model. Figure 2 shows the residuals from our current bestfit values to the X-ray data (and position angle from the radio images) using the anticorrelation model [3]. The model parameters are: precession phase, $\phi$, precession half-angle, $\theta$, the jet speed, $\beta$, and a time delay that indicates how advanced the X-ray measurements are relative to the optical.

\section{Jet Symmetry in SS 433}

\subsection{Jet Velocity Asymmetry}

There are clear differences between the fit in Figure 1 and the red- and blue-shifted data (c.f. the data points near HJD 2453595). The deviations are consistent in both the optical and X-ray 
data. Figure 2 shows the difference in terms of the modified model [3] jet velocity variations were required to adequately match the data $\left(\beta_{b}=0.04\right.$ and $\left.\beta_{r}=0.01\right)$.

There is also a rapid change in $\mathrm{z}$ visible in the blue-shifted jet at HJD 2453598 but no corresponding change in the red jet. To properly fit such changes, the model would require additional jet velocity variability on timescales of less than a day. Velocity variability on this scale would not have been observed previously because the data used to define the modified kinematic model consisted of optical spectra. The fragmentation of the optical data into bullets and the long persistence time (up to one week) of those optical bullets would make shorter timescale studies difficult or impossible. However, the X-ray jet emission has a much shorter persistence time (much less than one day) and does not appear to occur in discrete bullets, but rather in a semi-continuous source.

\subsection{Red-shifted vs Blue-shifted Line Strength}

The X-ray spectra show a clearly stronger blue-shifted component. There are spectra in which the red-shifted component is nearly undetected. In the optical as well, the red-shifted components often showed lower line strengths than the blue-shifted jets. In those cases, the weakest detected blue-shifted jet lines did not have corresponding detected red-shifted lines.

\section{Discussion}

We will be pursuing this data with the goal of providing a more complete description of the relativistic jets in the SS 433 system. The velocity asymmetries, rapid radial velocity variability, which could be caused by variation in jet velocity, jet-cone angle, or both, and the rapid position angle changes will be explored further

We will also be comparing the appearance times of the X-ray and optical data to determine at what point along the jet these emissions originate. Comparison with proper motions and position angles from the radio data will constrain the true physical motion of the jets.

Financial support was provided by the National Science Foundation through grant AST-0607432 (T.C.H.)

\section{References}

[1] J.I. Katz, S.F. Anderson, B. Margon, \& S.A. Grandi, Nodding motions of accretion rings and disks - A short-term period in SS 433, 1982, ApJ, 260, 780

[2] D.R. Gies, M.V. McSwain, R.L. Riddle, Z. Wang, P.J. Wiita, \& D.W. Wingert, The Spectral Components of SS 433, 2002, ApJ, 566, 1069

[3] K.M. Blundell \& M.G. Bowler,Jet Velocity in SS 433: Its Anticorrelation with Precession-Cone Angle and Dependence on Orbital Phase 2005, ApJL, 622, 129 